\title{
Increased DNA methylation, cellular senescence and premature epigenetic aging in guinea pigs and humans with tuberculosis
}

\author{
Carly A. Bobak ${ }^{1, *}$, Abhimanyu 2,3,4* , Harini Natarajan5, Tanmay Gandhi ${ }^{6,7}$, Sandra L. Grimm ${ }^{6,7}$, \\ Tomoki Nishiguchi ${ }^{2,3,4}$, Kent Koster ${ }^{8}$, Santiago Carrero Longlax ${ }^{2,3,4}$, Qiniso Dlamini ${ }^{9}$, Jacquiline \\ Kahari $^{9}$, Godwin Mtetwa9 ${ }^{9}$, Jeffrey D. Cirillo ${ }^{8}$, James O'Malley $^{1,10, \#, \text { Jane E. Hill }}{ }^{11, \#}$, Cristian \\ Coarfa ${ }^{6,7, \#}$, Andrew R. DiNardo $2,3,4, \#$ \\ ${ }^{1}$ Biomedical Data Science, Geisel School of Medicine, Dartmouth College, Hanover, NH 03755, USA \\ ${ }^{2}$ The Global Tuberculosis Program, Baylor College of Medicine, Houston, TX 77030, USA \\ ${ }^{3}$ William Shearer Center for Human Immunobiology, Texas Children's Hospital, Houston, TX 77030, USA \\ ${ }^{4}$ Immigrant and Global Health, Department of Pediatrics, Baylor College of Medicine, Houston, TX 77030, USA \\ ${ }^{5}$ Department of Microbiology and Immunology, Geisel School of Medicine at Dartmouth, Dartmouth College, \\ Hanover, NH 03755, USA \\ ${ }^{6}$ Dan L Duncan Comprehensive Cancer Center, Baylor College of Medicine, Houston, TX 77030, USA \\ ${ }^{7}$ Molecular and Cellular Biology Department, Baylor College of Medicine, Houston, TX 77030, USA \\ ${ }^{8}$ Department of Microbial Pathogenesis and Immunology, Texas A\&M University Health, Bryan, TX 77807, USA \\ ${ }^{9}$ Baylor-Swaziland Children's Foundation, Mbabane, Swaziland \\ ${ }^{10}$ The Dartmouth Institute, Dartmouth College, Hanover, NH 03755, USA \\ ${ }^{11}$ Department of Chemical and Biological Engineering, University of British Columbia, Vancouver, BC, Canada \\ *Co-first authors contributing equally \\ ${ }^{\#}$ Co-senior authors contributing equally
}

Correspondence to: Carly A. Bobak, Cristian Coarfa, Andrew R. DiNardo; email: carlybobak@dartmouth.edu, coarfa@bcm.edu, andrew.dinardo@bcm.edu

Keywords: tuberculosis, multi-cohort analysis, network analysis, DNA methylation, Cavia porcellus, senescence, DNA hypermethylation, epigenetic aging

Received: December 23, 2021 Accepted: February 22, $2022 \quad$ Published: March 7, 2022

Copyright: (c) 2022 Bobak et al. This is an open access article distributed under the terms of the Creative Commons Attribution License (CC BY 3.0), which permits unrestricted use, distribution, and reproduction in any medium, provided the original author and source are credited.

\section{ABSTRACT}

Background: Tuberculosis (TB) is the archetypical chronic infection, with patients having months of symptoms before diagnosis. In the two years after successful therapy, survivors of TB have a three-fold increased risk of death.

Methods: Guinea pigs were infected with Mycobacterium tuberculosis (Mtb) for 45 days, followed by RRBS DNA methylation analysis. In humans, network analysis of differentially expressed genes across three TB cohorts were visualized at the pathway-level. Serum levels of inflammation were measured by ELISA. Horvath (DNA methylation) and RNA-seq biological clocks were used to investigate shifts in chronological age among humans with TB.

Results: Guinea pigs with TB demonstrated DNA hypermethylation and showed system-level similarity to humans with TB ( $p$-value $=0.002)$. The transcriptome in TB in multiple cohorts was enriched for DNA methylation and cellular senescence. Senescence associated proteins CXCL9, CXCL10, and TNF were elevated in TB patients compared to healthy controls. Humans with TB demonstrate 12.7 years (95\% $\mathrm{Cl}: 7.5,21.9)$ and 14.38 years (95\% Cl: $10.23-18.53)$ of cellular aging as measured by epigenetic and gene expression based cellular clocks, respectively.

Conclusions: In both guinea pigs and humans, TB perturbs epigenetic processes, promoting premature cellular aging and inflammation, a plausible means to explain the long-term detrimental health outcomes after TB. 


\section{INTRODUCTION}

Tuberculosis (TB), caused by Mycobacterium tuberculosis $(\mathrm{Mtb})$, is responsible for about 1.4 million deaths worldwide each year [1]. TB is the archetypical chronic infection, with one-third of TB patients contending with the disease for a decade in the preantimicrobial era. Currently, TB patients have a median of three months of symptoms before diagnosis and retain a three-fold increased risk of mortality in the two years following successful therapy $[2,3]$. In the US, premature death after successful treatment for TB was associated with seven years of life lost [4]. In a recent meta-analysis of over forty-thousand TB cases, mortality during TB treatment was $7 \%$, and mortality in the two years after successful TB therapy was $16.9 \%[2]$.

Mouse models have demonstrated that in the setting of chronic infection, the epigenome rearranges to dampen host immunity and prevent host-inflicted immune pathology [5-7]. Specifically, the chronic antigenic stimulation results in histone deacetylation, histone methylation, and DNA hyper-methylation. Epigenetic modifications are a key process for the regulation of immune homeostasis as demonstrated in murine models of sepsis and chronic Lymphocytic choriomeningitis virus (LCMV), as well as human infections with HIV, TB schistosomiasis, and pneumonia [6, 8-11]. We recently demonstrated that $\mathrm{TB}$ patients develop DNA methylation changes similar to those seen in these animal models of chronic infection, with DNA hypermethylation of genes involved in IL2-STAT5, TNF$\mathrm{NF} \kappa \mathrm{B}$, and IL12-IFN $\gamma$ signaling pathways. Interestingly, these DNA hyper-methylation scars persist twelve months from initiation of antibiotics, six months from completion of successful TB therapy [8].

Our previous study in humans collected blood at the time of TB diagnosis, and therefore it is possible that these individuals already accumulated these detrimental epigenetic marks before TB. In this work using an established guinea pig (Cavia porcellus) model of TB, we clarify that TB induced DNA methylation changes that statistically overlapped with methylation changes observed in humans with TB. Thereafter, through untargeted network analysis of gene expression data, we demonstrated that TB induced gene expression changes that enriched for DNA methylation and other epigenetic processes in multiple cohorts, including people living with or without Human Immunodeficiency Virus (HIV), and in both adults and children. Network analysis of both gene expression and DNA methylation also confirmed that TB is associated with oxidative-stress induced senescence (OSIS) and the senescenceassociated secretory pathway (SASP). By analysis of proteins shown to be elevated in SASP in an independent cohort from Eswatini, we confirmed that TB patients have increased SASP-related proteins [12] as well as increased Epigenetic and RNA-based age, with a mean increase from chronological age of 12.7 and 14.4 years respectively and a hazard ratio (HR) of 2.89 in all-cause mortality. In combination, the guinea pig and human data provide evidence that TB induces DNA hypermethylation, premature cellular aging, and inflammation.

\section{RESULTS}

TB induced DNA hypermethylation in guinea pigs, recapitulating global and specific features of human TB

Guinea pigs (Cavia porcellus) are a standardized animal model for TB, with many features that replicate human disease [13]; therefore, we evaluated the DNA methylation differences between TB diseased and control animals $(n=4)$. Methylation status of DNA isolated from spleen and lung tissue was evaluated 45 days post-infection (Figure 1A). Compared to uninfected controls, guinea pigs with TB had DNA hypermethylation of 2339 genes $(80 \%$ of differentially methylated genes) in the spleen and 600 genes $(60 \%$ of differentially methylated genes) in the lung (over $10 \%$ methylation ratio change and $p<0.05$; Figure 1B; Supplementary Table 1). Genes critical to mycobacterial immunity, such as NFKB1, TYK2, RPTOR, IL1R1 and $T O X$, were hypermethylated in either lung or spleen (Figure 1C, 1D). Spleen and lung shared 244 differential hypermethylated genes (DHG) ( $p$-value of overlap $=1.24 \times 10^{-65}$, Figure 1D) and 256 hypermethylated pathways ( $p$-value of overlap $=3.84 \times$ $10^{-120}$, Figure 1E). The common hypermethylated pathways in the lungs and spleen of guinea pigs included cytokine signaling, calcium signaling, metabolism, PI3K-AKT signaling, and tyrosine kinase pathways (Figure 1F).

We previously described DNA hypermethylation in humans with TB [8]. When comparing the DNA hypermethylation changes between humans and guinea pigs with $\mathrm{TB}$, there was a statistically significant overlap with peripheral blood in human CD4, CD8 and CD14 cells (Figure 2A, Kolmogorov-Smirnov $p$-value $=$ 0.002 ). Both humans and guinea pigs with $\mathrm{TB}$ demonstrated DNA hypermethylation of genes involved in pathways for the immune system, MAPK, tyrosine kinase, mTOR, calcium signaling, metabolism, and chromatin modifying enzymes (Figure 2B; Supplementary Table 2). Similarly, both humans and guinea pigs shared epigenetic changes in genes targeted by immune-related transcription factors (TF), including 
NFKBIA, TCF7, CIITA, MYC, NFAT and DNMT1/3A (Supplementary Figure 1; Supplementary Table 3). The TF were predicted to be regulating multiple genes across the groups (Supplementary Figure 1), establishing a systems-level overlap between human TB and guinea pigs. We previously demonstrated that TBassociated DNA hypermethylation in humans statistically overlapped with immune exhaustion, as induced by chronic infection with clone 13 LCMV in mice $[8,10,11]$. This was further validated by the DNA hypermethylation changes in guinea pigs enriching for targets of transcription factors, such as NFAT, MYC, TCF7, and NFKBIA (Supplementary Figure 1; Supplementary Table 3), which have been shown to lead to an immune inhibitory phenotype [14]. Combined gene and pathway analysis, using Metascape [15], demonstrated an interaction network enriched between humans and guinea pigs, including pathways for MAPK signaling, endocytosis, antigen presentation, calcium transport, cell adhesion, O-glycosylation of proteins, and chromatin modifying enzymes (Supplementary Figure 2).

A

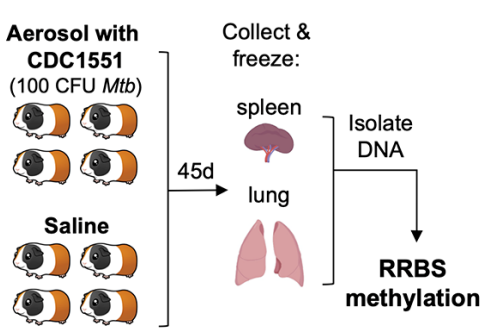

D

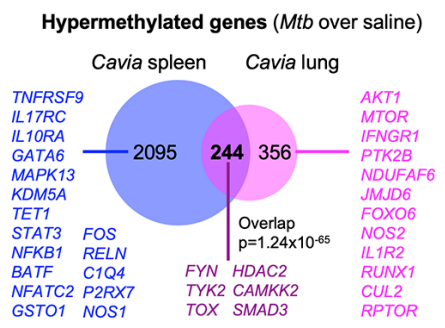

\section{B}

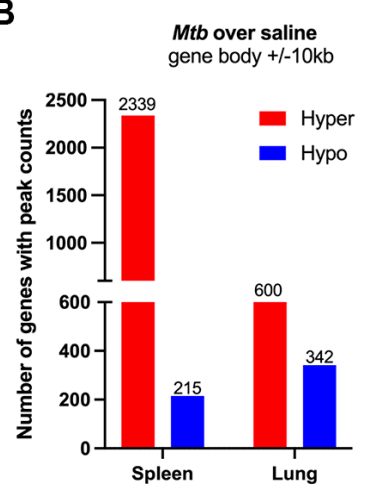

E

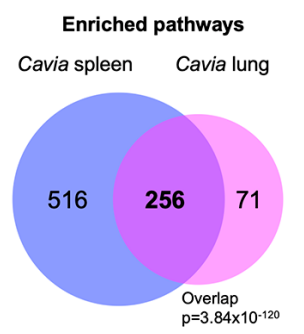

Enrichment of epigenetic regulators in human TB gene expression

To orthogonally validate the results, gene expression studies were also evaluated. Three datasets were evaluated based on their differing epidemiology. GSE42834 included adults with TB that excluded people living with HIV [16]; GSE37250 included adults with TB also living with HIV [17]; GSE39940 included children with TB [18]. The expression array datasets were collected from studies aiming to identify gene signatures that discriminated TB both from healthy controls as well as from other diseases, such as sarcoidosis, pneumonia, cancer, and other diseases that can mimic the clinical presentation of TB.

Differentially expressed genes and pathways were visualized as a network of pathway-level results, with major pathway groups derived from an enrichment map (Figure 3). Subnetworks relevant to TB disease are shown in Figure $3 \mathrm{~A}$, the full network is available in [19].
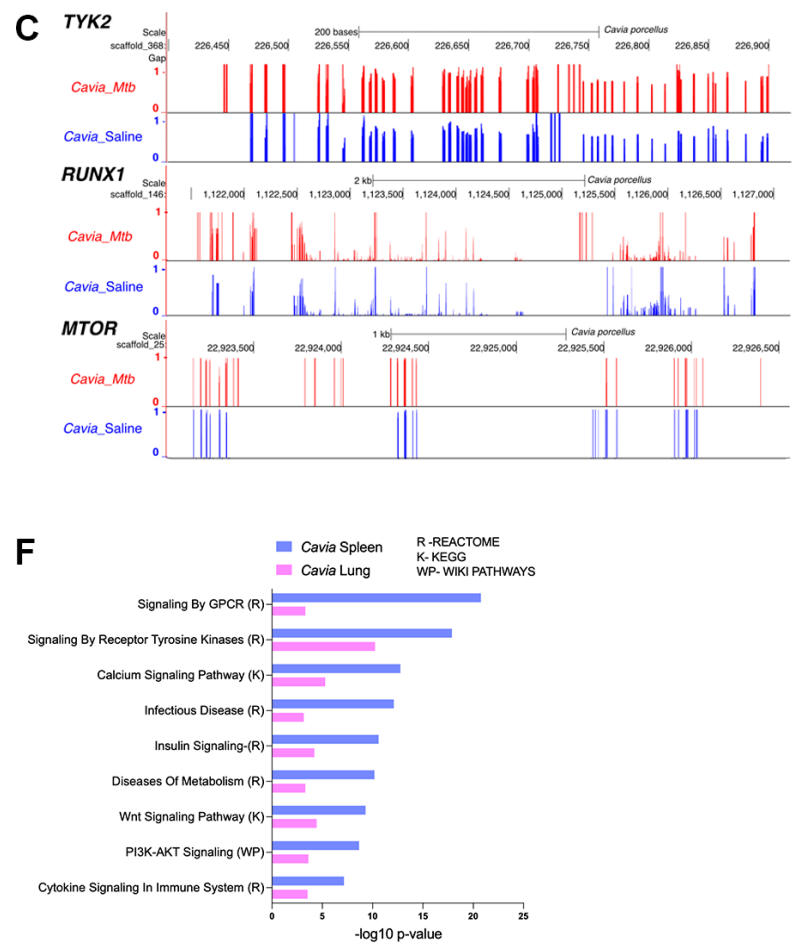

Figure 1. Guinea pigs (Cavia porcellus) with TB exhibited DNA hypermethylation. (A) Infection experimental design; guinea pigs were infected with $100 \mathrm{CFU}$ of Mtb CDC1551. Forty-five days later, spleen and lungs were removed with DNA methylation evaluated by RRBS. (B) Cavia with TB have DNA hypermethylation in lung and spleen when compared to uninfected controls. The number of genes with hypermethylation (red) or hypomethylation (blue) are plotted for each tissue (within 10kb from DMRs). (C) Genome browser (UCSC) view of a few key hypermethylated genes in Cavia with TB (red bars) as compared to non-infected "Saline" (blue bars). The bar plots represent methylation values from a scale of ' 0 ' unmethylated (black horizontal axis) to ' 1 ' fully methylated. Overall mean values combining both spleen and lungs are plotted. The Cavia scaffold position after alignment is indicated on top for each gene. (D) Shared and unique hypermethylated genes between lung and spleen. (E) Overlap of enriched pathways between Cavia spleen and lung (based on KEGG, Reactome, and Wikipathways) using hypermethylated genes. (F) Selected common pathways relevant to TB disease with their $-\log 10$ $p$-value of enrichment. 
(TB over healthy)

\section{Human CD4}

\begin{tabular}{|c|c|c|}
\hline 708 & 813 & Overlap \\
\hline 144 & 39 & $\begin{array}{l}\text { (S) } p=3.68 \times 10^{-6} \\
\text { (L) } p=0.0263\end{array}$ \\
\hline
\end{tabular}

Human CD8

$\begin{array}{lcl}7029 & 7774 & \text { Overlap } \\ 1037 & 292 & \begin{array}{l}\text { (S) } p=0.6074 \\ \text { (L) } p=0.0225\end{array} \\ 1302 & 308 & \\ \text { Cavia spleen } & \text { Cavia lung } & \end{array}$

Human CD14
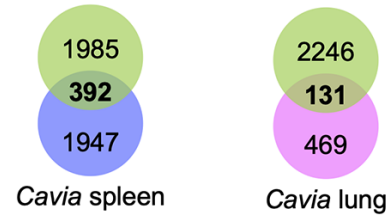

\section{Overlap}

\begin{tabular}{|c|c|c|c|c|c|}
\hline & \multicolumn{3}{|c|}{ Human } & \multicolumn{2}{|c|}{ Cavia } \\
\hline Signaling by receptor tyrosine kinases $(R)$ & 7.1 & 48.8 & 12.9 & 15.7 & 8.1 \\
\hline Calcium signaling pathway $(\mathrm{K})$ & 7.3 & 19.5 & 12.3 & 11.2 & 3.8 \\
\hline MAPK signaling pathway (WP) & 3.6 & 26.4 & 6.6 & 10.2 & \\
\hline Infectious disease $(R)$ & 2.3 & 21.2 & 6.4 & 10.2 & 2.2 \\
\hline Insulin signaling (WP) & 1.5 & 15.1 & 8.3 & 8.6 & 2.9 \\
\hline Diseases of metabolism $(R)$ & 1.9 & 11.1 & 3.1 & 8.4 & 2.3 \\
\hline PI3K/AKT signaling pathway (WP)- & 7.8 & 31.5 & 6.4 & 7.0 & 2.6 \\
\hline Cytokine signaling in immune system $(R)$ & 1.9 & 32.5 & 10.2 & 5.7 & 2.4 \\
\hline Chromatin modifying enzymes $(R)$ & & 4.2 & 1.9 & 5.1 & \\
\hline Chemokine signaling pathway $(\mathrm{K})$ & 2.1 & 13.1 & 4.0 & 3.4 & 2.3 \\
\hline MTOR signaling pathway $(\mathrm{K})$ & & 5.3 & 1.4 & 3.1 & 1.7 \\
\hline Interferon type I signaling pathways (WP & & 5.2 & 2.6 & 2.2 & 2.2 \\
\hline
\end{tabular}

(S) $p=3.95 \times 10^{-8}$

(L) $p=1.62 \times 10^{-9}$

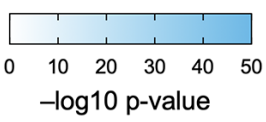

$-\log 10 \mathrm{p}$-value

Figure 2. Guinea pigs (Cavia porcellus) with TB exhibited systems-levels similarity with humans with TB. (A) Venn diagrams depicting the overlap between genes with DNA hypermethylation in guinea pig spleen (blue circle), lungs (pink circle) and humans with TB (CD4, yellow circle; CD8, blue circle; CD14, green circle). The $p$-value of overlap are shown on the side for 'S': Spleen; 'L": Lung. (B) Pathway enrichment analysis (MsigDB GSEA) demonstrating overlap in hypermethylated pathways in humans (light grey bars) and guinea pigs (dark grey bars) with TB. The box colors demonstrate $-\log 10 p$-value of enrichment, with darker shades of blue indicating significance, with the $-\log 10 p$-values written in the square. Selected TB-relevant pathways are depicted.

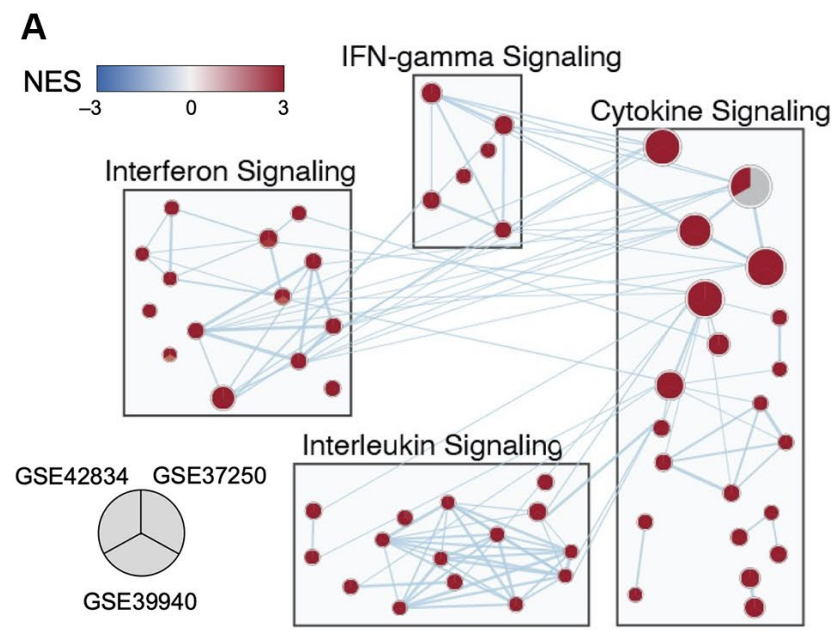

B Epigenetic Regulation

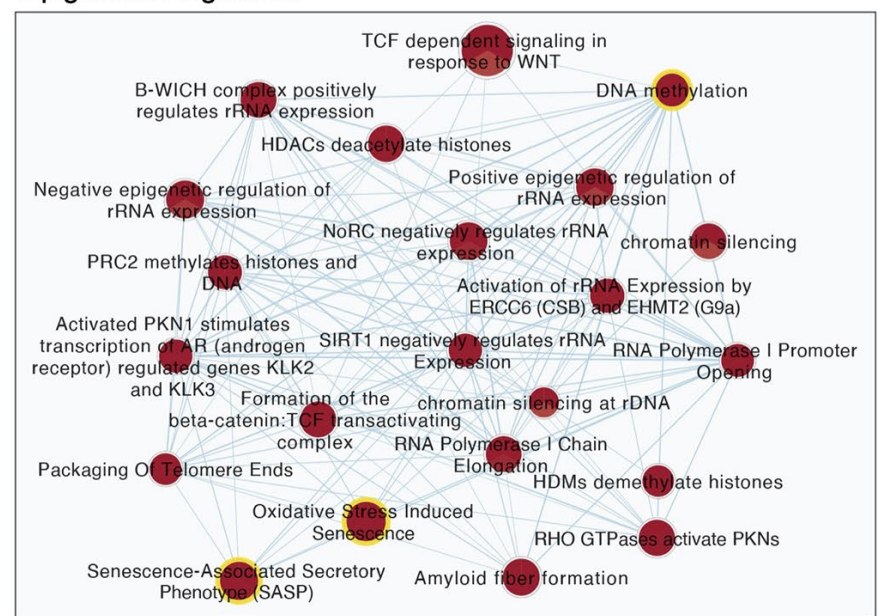

Figure 3. Multi-cohort transcriptomic analyses corroborated a role for epigenetic regulation in TB. (A) Selected subnetworks of enriched pathways associated with active TB diagnosis from three transcriptomic datasets. (B) Zoom-in of the Epigenetic Regulation Subcluster. Each node in the network represents an annotated gene set. Each node is a pie-chart corresponding to the normalized enrichment score (NES) for each dataset. Only nodes with a false discovery rate (FDR) $q$-value $<0.01$ in at least one dataset are depicted. Edges represent the overlap of genes between gene sets, where only overlaps $>0.55$ are visualized. The color of each pie on the map indicates the NES (blue for negative NES, and red for positive NES). The size of each node is proportional to the size of the gene sets. 
An abundance of genes involved in modifying the epigenome were differentially expressed in the discovery datasets, including regulators of acetylation such as the histone deacetylases (HDAC3, 1,5), the NAD-dependent deacetylases Sirtuins (SIRT1,2,4,5), regulators of methylation such as DNA methyltransferases $(D N M T 3 A)$, lysine demethylases $(K D M 6 B)$, and chromatin modifiers such as the polycomb repressor complex components EZH2 and SUZ12 (Supplementary Figure 3). Previous studies have demonstrated that epigenetic changes can be triggered by oxidative stress and senescence-associated pathways [20-23] which were also enriched in all three TB gene expression datasets (Figure 3B).

Within the epigenetic regulation network, the DNA methylation node is a hub with 20 connections to other gene sets (degree $=20$ ), including the oxidative stressinduced senescence and senescence-associated phenotype (Figure 3B). Across the three datasets, gene set enrichment analysis (GSEA) for DNA methylation genes had normalized enrichment scores (NES) of $2.10,2.32$, and 1.89 (adjusted $p$-value of $1.9 \times 10^{-5}$,
$3.9 \times 10^{-8}$, and $6.0 \times 10^{-4}$ ) for GSE42834, GSE37250, and GSE39940 respectively. From healthy controls, there was an increase in the DNA methylation summed $\mathrm{Z}$-score (Figure 4A). The most notable differences in signature Z-score occurred between samples from patients with TB and healthy controls or people with asymptomatic $M t b$ infection $\left(p=1.7 \times 10^{-11}\right.$, < $2.9 \times 10^{-26}, 3.5 \times 10^{-14}$ in GSE42834, GSE37250, and GSE39940 respectively). We observed graded separation comparing $\mathrm{TB}$ disease to other diseases across the three distinct cohorts, substantiating that DNA methylation-associated gene changes due to TB disease is reflective of prior work in other chronic infections (Supplementary Figure 4) [5, 7]. The summed z-score for SASP and OSIS also was increased in TB patients compared to healthy controls or asymptomatic $M t b$ infected controls $\left(p=1.0 \times 10^{-14}\right.$, $7.3 \times 10^{-38}, 4.4 \times 10^{-14}$ in the SASP pathway, $p=$ $1.3 \times 10^{-14}, 6.8 \times 10^{-32}, 7.5 \times 10^{-16}$ in the OSIS pathway for GSE42834, GSE37250, GSE39940 respectively; Figure 4B-4C). Correlation of DNA methylation, SASP, and OSIS pathway Z-scores was observed across all datasets (DNA methylation and OSIS $r=0.88,0.96$,
A
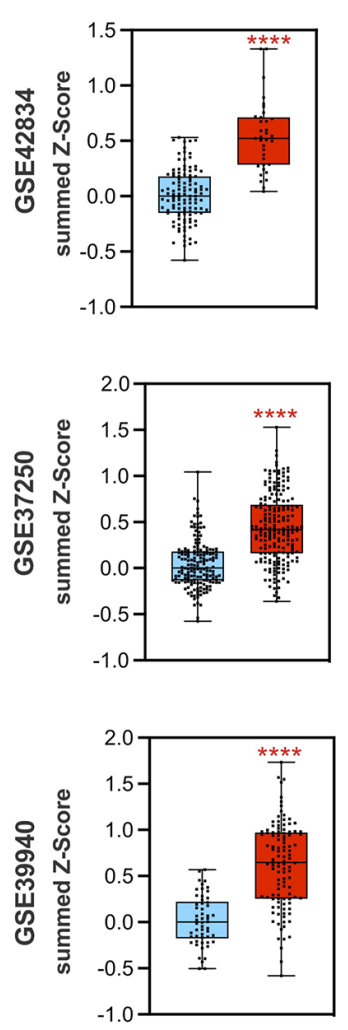
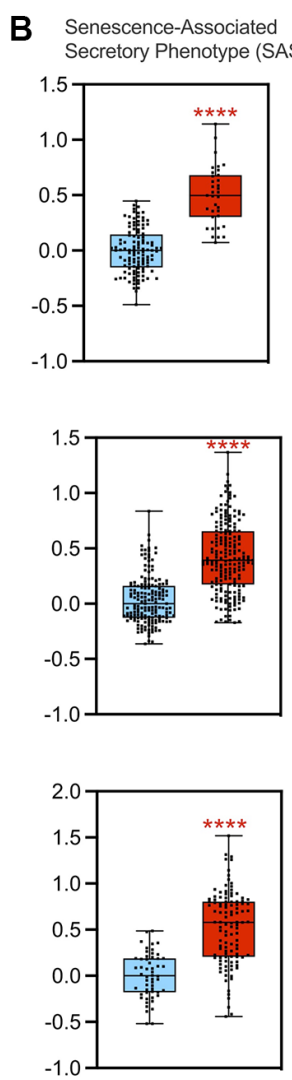
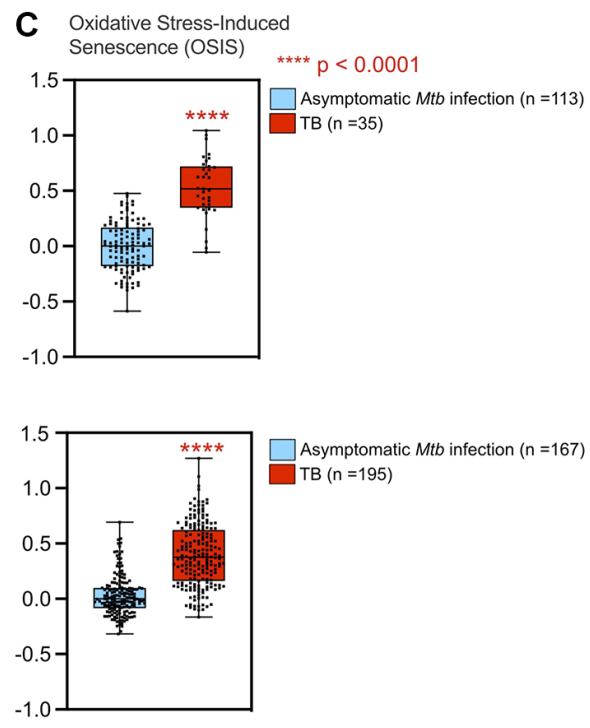

$\square$ Asymptomatic Mtb infection ( $n=167)$ $\square$ TB $(n=195)$

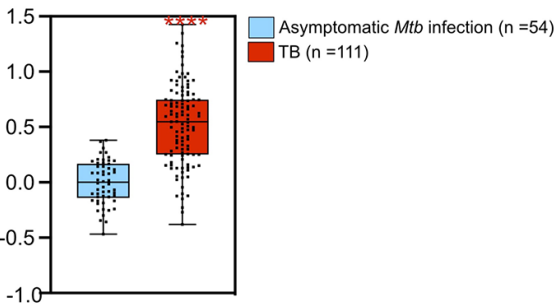

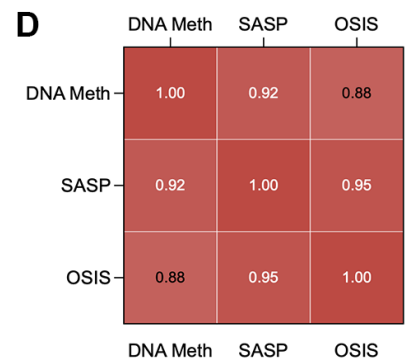
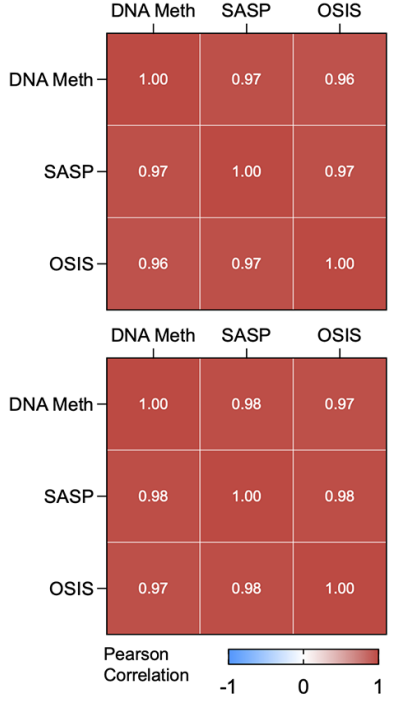

Figure 4. DNA methylation and cellular senescence genes are increased and correlated in TB. A summed z-score for gene expression from each patient was assessed for pathways including DNA methylation (A), SASP (B), and OSIS (C), with all three studies demonstrating increased summed z-scores in TB patients (red box plot) as compared to controls (blue box plot). $P$-values from a Wilcoxon rank sum test are indicated by asterisks. (D) DNA methylation correlated with senescence pathways using Pearson correlation for GSE42834, GSE37250, and GSE39940 respectively. Abbreviations: SASP: Senescence-associated secretory phenotype; OSIS: Oxidative stress-induced senescence. 
and 0.97; DNA methylation and SASP $\mathrm{r}=0.92,0.97$, 0.98; OSIS and SASP $\mathrm{r}=0.95,0.97,0.98$ for GSE42834, GSE32750, GSE39940 respectively; Figure 4D).

\section{TB induced premature epigenetic aging}

In both humans and guinea pigs with $\mathrm{TB}$, genes enriching for the SASP and OSIS pathways were hypermethylated compared to controls (Figure 5A). TB patients demonstrated a statistically significant enrichment of hypermethylated genes previously identified to have closed chromatin as part of the normal aging process [21] (NES $=1.47, p=2.93 \times 10^{-9}$; Figure $5 \mathrm{~B})$. At the protein level, in an independent cohort from Eswatini, TB patients demonstrated an increase in CXCL9, CXCL10, and TNFa, all components of the SASP (Figure 5C). Considering these findings, and the fact that aging is associated with increased DNA methylation, increased circulating senescence associated inflammation, and oxidative stress, we compared the biological age against the chronological age using two distinct biological clocks. The "Horvath clock" calculates biological age based on DNA methylation and has previously identified that HIV induced premature cellular aging [24, 25]. When the Horvath clock was applied to TB patients, there was an average increase in the DNA methylation age by 12.7 years above the chronological age (Figure 5D; Wilcoxon $p<0.0001,95 \%$ CI (7.5-21.9 years)). This increased epigenetic age persisted at least 12 months from TB diagnosis (6 months after the completion of successful therapy; Figure 5D). To evaluate cellular aging using an orthogonal approach, a recently developed transcriptomic (RNA-sequencing based) clock 'RNAAgeCalc' was applied to an RNA-seq dataset (GSE107993; Figure 5E) [26]. Compared to healthy contacts and patients with asymptomatic $M t b$ infection (also known as latent Mtb infection; LTBI), the transcriptomic clock of TB patients was increased 14.38 years $(95 \%$ CI: $10.22-18.53)$ over the chronological age $(p<0.0001$, ANOVA with Tukey's multiple comparison); Figure 5E). Moreover, individuals who were initially asymptomatic, but progressed to active TB disease within 6 months also demonstrated an increased transcriptomic clock of 4.83 years (95\% CI: 0.10-9.56), which was significantly higher than healthy contacts and patients with asymptomatic $M t b$ infection ( $p=0.0115$, ANOVA with Tukey's multiple comparison; Figure 5E). DNA methylation has been shown to be a strong predictor of all-cause mortality [27], including an increase in Horvath epigenetic age. Considering the Horvath age increase of 12.7 years, TB cases computed as per Zhang et al. [27] had an increased hazard ratio (HR) of 2.89 (95\% CI: $2.40-3.12)$ in all-cause mortality.

\section{DISCUSSION}

At the completion of apparently successful therapy, TB patients have a nearly 3-fold increased risk of death due to unknown reasons [2, 4]. Our previous work demonstrated that TB patients exhibited DNA hypermethylation associated with decreased immune responsiveness and that the DNA hypermethylation marks did not normalize six months after the completion of successful TB therapy [8]. Using a guinea pig model, this study corroborates that TB induces DNA hypermethylation marks and that the epigenetic perturbations in guinea pigs recapitulated human disease. Further, at transcriptomic and epigenomic levels, both guinea pigs and humans with TB enriched for senescence and oxidative stress induced senescence pathways, with humans afflicted with TB demonstrating more than a decade of premature cellular aging by both epigenetic and transcriptomic clocks. Increases in DNA hypermethylation and cellular senescence are plausible mechanisms for the long-term increased risk of death in TB patients and, as such, they need to be prospectively evaluated.

In order to temper exuberant, pathologic immunity in the setting of chronic or severe infection, epigenetic mechanisms dampen both adaptive and innate immunity to prevent collateral host tissue destruction $[5,6,28]$. Unfortunately, these epigenetic scars are long-lived, thereby increasing the risk for secondary infections [7]. Both severe and chronic infections perturb host epigenetic regulation, thereby suppressing host immunity [29-31]. HIV also perturbs host epigenetic regulation, resulting in long-lasting DNA methylation changes in IL-2, PD1, and the regulation of IFN- $\gamma$ $[32,33]$.

Previously, it was unclear if individuals with DNA hypermethylation were at increased risk of progressing to $\mathrm{TB}$, or if $\mathrm{TB}$ itself was inducing DNA hypermethylation. While the options are not mutually exclusive, the guinea pig studies lucidly demonstrated that TB induces DNA hypermethylation. This is also supported by the orthogonal gene expression analysis, demonstrating an increase in genes related to DNA methylation among TB patients. This does not exclude the possibility that individuals with increased DNA methylation are at increased risk of progression to TB. In fact, the gene expression analysis suggests that multiple diseases induce DNA methylation perturbations. Both schistosomiasis and HIV induced DNA hypermethylation and both are associated with increased risk of TB disease progression [33, 34]. Further, compared to asymptomatic controls that stayed healthy, individuals that were originally asymptomatic, 
but then progressed to overt TB disease, had an increase in premature aging as calculated by the RNA clock. Plausibly, comorbidities like air pollution, smoking, schistosomiasis, and HIV result in DNA hypermethylation, suppressing the host immune response, and therefore increasing the risk of $\mathrm{TB}$
A

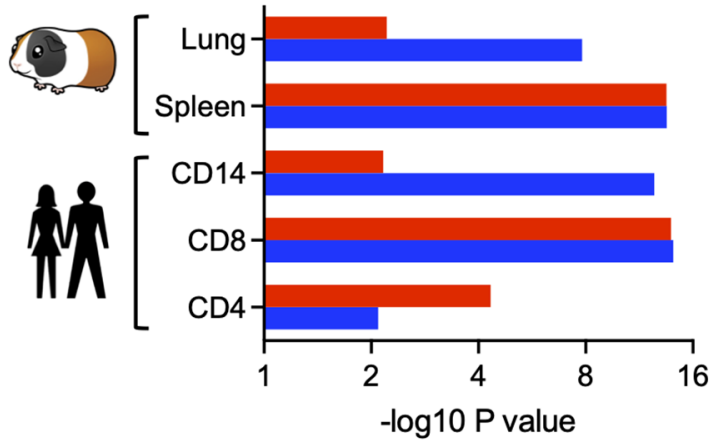

Senescence Associated Secretory Phenotype

Oxidative Stress Induced Senescence

\section{B TB-induced Age-associated hyper-methylation closed UCAR}

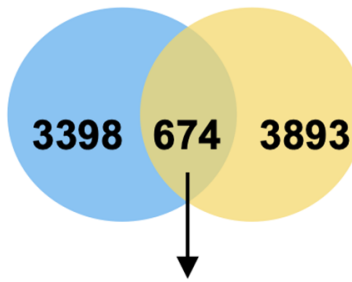

Common genes: Common Pathways:

STAT1

STAT4

NFKB

RPTOR
PI3K-mTOR

IFN signaling

TNF-NFKB signaling

IL6-JAK-STAT signaling

\section{C}

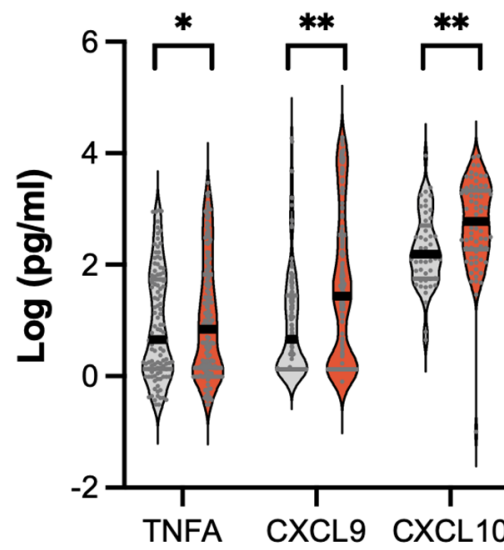

D

$$
\begin{gathered}
\text { Chronologic Age (CA) versus } \\
\text { Epigenetic Age (EA) }
\end{gathered}
$$

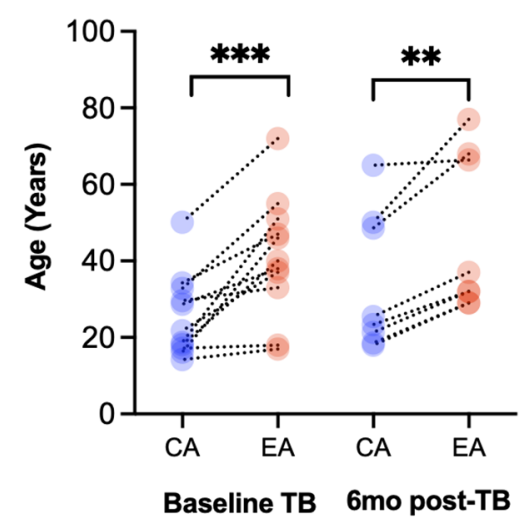

E
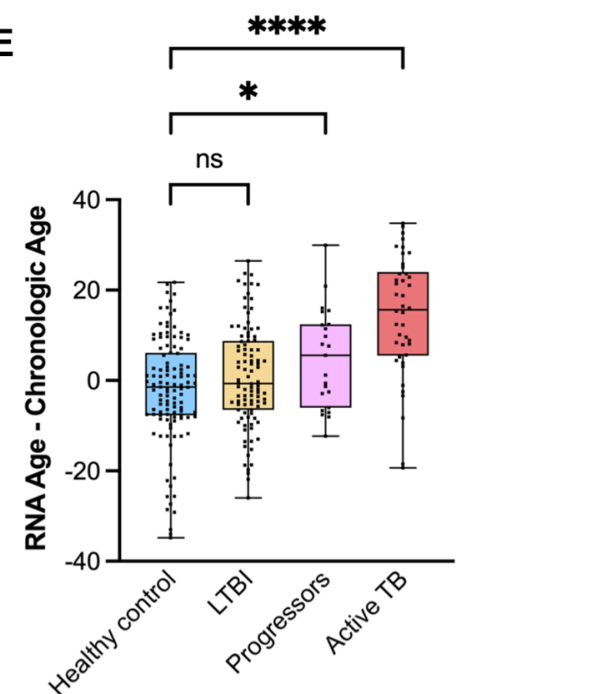

Figure 5. TB induced cellular senescence and premature cellular aging. (A) Humans and guinea pigs with TB demonstrated DNA hypermethylation gene changes that enriched for the SASP and OSIS pathways (Reactome overrepresentation $p$-values). (B) Hypermethylated genes in CD8+ T cells from patients with TB statistically overlapped with old age-associated closed chromatin conformation changes. (C) Multiplex ELISA of senescence associated proteins in patients with TB compared to healthy controls. (D) Epigenetic age (using the Horvath DNA methylation clock) is increased as compared to chronological age in TB patients at baseline and 6 months after the completion of successful anti-TB therapy. (E) Difference between chronological age and biological age using the RNA age calculator demonstrates an increase in TB patients compared to healthy controls (one-way ANOVA with Tukey's multiple comparison). 
disease progression. Future experiments will need to evaluate if preceding unrelated infection would further predispose risk for TB progression due to epigenetic perturbations.

After successful therapy for pneumonia, sepsis, or TB, there remains an increased risk of mortality [4, 35-37]. A recent retrospective study examining 2522 TB patients in the United States calculated an average of 7.0 (95\% CI: 5.5-8.4) years of potential life lost despite apparently successful therapy [4]. Similarly, we calculated a nearly 3 -fold risk $(\mathrm{HR}=2.89)$ in all-cause mortality in TB patients using predictors described by Zhang et al. [27]. The observations that cellular senescence is enriched in both gene expression and DNA methylation datasets of patients with $\mathrm{TB}$, and the correlation between methylation and senescence need to be mechanistically explored to identify if the $>10$ years of premature cellular aging is reversible. Additional evidence supporting TB induced "inflammaging" includes elevations in plasma levels of SASP markers such as TNF $\alpha$, CXCL9, and CXCL10, providing additional evidence that long-term health and mortality is negatively impacted by the development of $\mathrm{TB}$ disease. This phenomenon has previously been observed in people living with HIV in a study conducted by Horvath et al. [24]. The increase in pathways of DNA methylation, oxidative stress induced cellular senescence, and senescence associated secretory phenotype summed z-scores, and correlation between these pathways, suggests there are common epigenetic perturbations that occur with chronic inflammation, offering optimism that there may be common pathways to remediate these changes. Further understanding of the role of accelerated aging in TB patients may be also elucidated by decreased telomere length and increased mitochondrial DNA copy number in TB patients [38].

An interesting observation was the lower number of changes observed in the lungs as compared to the spleen in the guinea pigs. Although we see a significant overlap between post-infection methylation changes between the two organs, our analysis reveals a tissuespecific response to pathogens, which may partially be explained by the distinct cell type composition of the two tissues.

While the results of the DNA methylation studies were limited by the small sample sizes in the guinea pigs, this would still allow for the observation of large effect sizes. Further, the findings were corroborated using orthogonal transcriptomic and protein analyses. We also see statistically significant overlap with the previous DNA hypermethylation findings in human studies suggesting that even the small size of the guinea pig model was able to capture major meaningful perturbations in the epigenome. Future examinations will need to validate these observations in larger numbers in both guinea pigs and humans, with particular focus on the commonly perturbed pathways like PI3K/AKT or MTOR signaling. Experiments suggest that immune inhibitory epigenetic marks were triggered by specific events (NFAT homodimerization, intracellular metabolic shifts) that resulted in histone deacetylation, DNA hypermethylation, and chromatin closing [39, 40]. A longitudinal approach should be considered to examine if increases in DNA methylation or premature aging are associated with increased risk of TB disease progression and, vice versa, if DNA methylation and premature aging improve after successful therapy.

\section{CONCLUSIONS}

This work, using multiple cohorts, multiple tissue types, and both transcriptomic and DNA methylation sequencing, provides evidence that $\mathrm{TB}$ induced perturbations in epigenetic regulation, specifically in DNA methylation that correlated with oxidative stress induced senescence and was associated with premature cellular aging, measured by both epigenomic and transcriptomic based clocks. These processes were conserved across both guinea pigs and humans, indicating that a guinea pig (Cavia porcellus) model may be appropriate for further mechanistic research. The TB induced premature aging is a plausible mechanism for increased risk of death that occurs after successful therapy for TB and needs to be prospectively evaluated.

\section{MATERIALS AND METHODS}

\section{Animal aerosol challenge, determination of bacterial load, and DNA extraction}

All guinea pig experiments were reviewed and approved by the Institutional Care and Use Committee at Texas A\&M University in compliance with the National Institute of Health guidelines, as described in the Guide for the Care and Use of Laboratory Animals. Eight pathogen-free female Harley guinea pigs (250$300 \mathrm{~g}$ ) were obtained from Charles River Laboratories. The animals were maintained with commercial diet and water ad libitum. The guinea pigs were divided into two groups of four, one group underwent aerosol infection with Mtb strain CDC1551, grown as described previously [41, 42] and washed with saline and suspended at an $\mathrm{OD}_{600}$ of 0.1 prior to nebulization using a Madison chamber, as described previously [41, 43]. The other group was used as a control without any infection procedure. Initial colony forming units (CFU) in the lungs were $\sim 100 \mathrm{CFU}$. The average number of CFU present in the entire lungs at 45 days was $4.2 \pm$ 
$0.7 \times 10^{6} \mathrm{CFU}$, the CFU plateaued just after 45 days post-infection, which was hence chosen as the time point at which humane euthanasia was performed with pentobarbital (FatalPlus). At necropsy, organs were collected and frozen at $-80^{\circ} \mathrm{C}$ until use. DNA was isolated from organs using the DNeasy blood and tissue kit (Qiagen Inc.), followed by nucleic acid quantity determination with a Qubit fluorometer using the dsDNA BR assay kit (Thermo Fischer Scientific, Waltham, MS, USA).

\section{Quantification of DNA methylation in guinea pigs and humans}

DNA methylation from the spleen and lung of the control and TB guinea pigs was measured using reduced representation bisulfite sequencing (RRBS) as previously described [44, 45] to map the methylated cytosines in the DNA obtained from spleen and lung from infected and uninfected Cavia porcellus, as described in the previous section. Library preparation was performed using Ovation RRBS Methyl-Seq System kit (NuGEN Technologies, Inc, Redwood City, California). In brief, $100 \mathrm{ng}$ of genomic DNA was digested with $M s p I$, and Illumina-compatible cytosinemethylated adaptors were ligated to the enzymedigested DNA. Size-selected fragments were bisulfiteconverted, and library preparation was done by PCR amplification, and subsequently sequenced in a HiSeq3000 instrument at the MDACC Epigenomics Profiling Core Facility. RRBS sequencing reads were aligned to Cavia reference genome and DNA methylation ratios at $\mathrm{CpGs}$ were called using Bismark v0.7.11 [46]. Next, differentially methylated $\mathrm{CpG}$ sites (DMCs) and differentially methylated regions (DMRs) between infected and uninfected Cavia were identified using BS-seq [47]. DMR-associated genes were determined using BEDTOOLS [48] and a window of 10,000 base pairs around coding gene bodies.

Peripheral blood mononuclear cells (PBMCs) from human subject samples, previously published, underwent DNA isolation using the Qiagen DNeasy kit, followed by bisulfite conversion [8]. DNA methylation was determined by the Illumina MethylEPIC array and was deposited in the NCBI Gene expression omnibus (GSE145714). The gene list was also published as a supplementary table, from which the information was obtained for the overlap analyses [8].

\section{Functional enrichment analysis for guinea pig and human methylation}

To evaluate gene or pathway overlaps Venn diagrams were obtained using the online portal (http://bioinformatics.psb.ugent.be/webtools/Venn/).
Gene set enrichment analysis was carried out using the hypergeometric distribution as implemented by the MsigDB online tool (http://www.gsea-msigdb.org/) against the following gene collections including hallmark, KEGG, Reactome and WikiPathways to compute overlaps with an FDR cut-off $<0.05$ for the significant genes from the guinea pig and human methylation datasets [49]. Overall system level similarly was compared using a two-sided KolmogorovSmirnov test, where overlap $p$-values were compared to the null hypothesis that they follow a uniform distribution. In addition, targets of transcription factor (TFT) overlaps were also computed for all lists using the same portal. Meta-pathway analysis was used to find gene and simultaneous pathway level overlap between the human and the guinea pig datasets through Metascape online pathway analysis portal (https:// metascape.org/gp/index.html\#/main/step1) [15]. Overrepresentation analysis for OSIS and SASP was carried out using the analysis option of the Reactome database, where the hypermethylated genes from humans and guinea pigs were used as input. The protein-protein interaction (PPI) network was generated using MCODE algorithm to identify enriched clusters, represented as a merged network visualized using Cytoscape [50, 51]. The network was then annotated using gene ontology (GO) enrichment analysis-as described by Zhou et al. [15] The bar graphs were plotted using GraphPad Prism (version 9.1.0) for macOS [52].

\section{Quantification of the serum cytokines in a TB cohort}

The plasma samples were obtained from a previously described cohort of adults with TB symptoms and microbiologically confirmed pulmonary TB (by culture and/or GeneXpert) from Eswatini. They were compared to with their asymptomatic, healthy household contacts who remained asymptomatic for 12 months after initial exposure [8]. The serum was evaluated by using nine soluble markers of senescence associated secretory phenotype as defined previously [53, 54], including IL15 $(\mathrm{TB}=40, \mathrm{HC}=39)$, TNFA $(\mathrm{TB}=152, \mathrm{HC}=$ $111)$, IL1B $(\mathrm{TB}=152, \mathrm{HC}=111), \mathrm{CXCL} 10(\mathrm{~TB}=70$, $\mathrm{HC}=49)$, IL6 $(\mathrm{TB}=70, \mathrm{HC}=49)$, CXCL9 $(\mathrm{TB}=152$, $\mathrm{HC}=111), \operatorname{IL} 7(\mathrm{~TB}=112, \mathrm{HC}=72), \mathrm{GMCSF}(\mathrm{TB}=$ $82, \mathrm{HC}=62)$, IL1A $(\mathrm{TB}=82, \mathrm{HC}=62)$ using a customized bead-based multiplex assay (LEGENDplex kits, BioLegend), according to manufacturer's instruction. Briefly, the human plasma was diluted 1:1 and incubated with APC-conjugated capture beads, specific to each analyte being measured, supplied with the kit. Known standards for each analyte were also incubated in duplicate. Biotinylated detection antibodies were added which form capture bead-analyte-detection antibody sandwiches. Streptavidin-phycoerythrin (SA-PE) was subsequently added, providing fluorescent 
signal with intensities in proportion to the amount of bound analyte. For each bead population, the PE signal fluorescence intensity was quantified using a flow cytometer. The concentration of a particular analyte was determined based the known standard curve using the LEGENDplex data analysis software.

\section{Gene expression cohort selection}

Gene expression array datasets were downloaded from the Gene Expression Omnibus (GEO) hosted by the National Center for Biotechnology Information (NCBI). Search terms to identify datasets included 'Tuberculosis' and 'TB' and were limited to whole blood samples. We selected three datasets based on the heterogeneity of the epidemiology. The first cohort included only adults and excluded HIV positive patients [16]. The second cohort included adults, both people with and those without HIV co-infection [17]. Finally, the last cohort included children, with and without HIV co-infection [18]. All three datasets were analyzed using the Illumina HumanHT12v4 bead chip. A total of 1152 samples were used in analysis. These studies were designed to identify a novel TB diagnostic test, and therefore they included diseases that present similar to $\mathrm{TB}$, including other pneumonias, sarcoidosis, cancer, and other infections.

\section{Analysis of expression data}

Analyses were conducted in $\mathrm{R}$ version 4.0.3. Normalized data were downloaded using the 'GEOquery' package, and checked to ensure that values were mean-centered and $\log _{2}$ transformed [55]. Differential expression (DE) analysis of genes was conducted using the 'limma' package in $\mathrm{R}$ to calculate the empirical Bayes (moderated) pooled variance for each gene where active TB was contrasted to all the other disease classes as a one-vs.-rest problem. DE analysis identified genes that were significantly associated (either positively or negatively) with the outcome of interest, in this case active TB disease.

The $\log _{2}$ fold change (FC) and $p$-values from the DE analysis were used to calculate scores and rank genes for Gene Set Enrichment Analysis (GSEA) using

$$
\text { Rank }=\operatorname{sign}\left(\log _{2}(F C) \times(-1) \log _{10}(p)\right.
$$

as suggested in [56]. These ranks were input to GSEA Desktop v3.0 to identify which gene sets were enriched or depleted in each of the three data sets [57]. The Gene Ontology (GO) process collection of gene sets was downloaded from the Molecular Signature Database (MSigDB) [49]. GSEA compared gene sets and genes which were close to the top or the bottom of the ranked list to determine whether the gene set was over-represented. Each gene set was given a Normalized Enrichment Score (NES) [57]. The output after running GSEA was used to create an EnrichmentMap in Cytoscape v.3.6.0 [50, 58]. Subnetworks were identified using AutoAnnotate [59] and those related to epigenetic regulation are presented.

We evaluated the DNA methylation, oxidative stress induced cellular senescence, and senescence associated secretory phenotype genes using a pathway z-score, calculated as the as the difference of the mean of upregulated and the mean of downregulated genes contained in each pathway and measured in each dataset, as suggested in [60]. We centered pathway scores on the median value observed in the nonasymptomatic control group in each dataset. A Wilcoxon rank sum test was used to test if these pathway scores were significantly different between TB and healthy controls or other diseases [61]. We calculated the correlation between the observed z-scores of each of the epigenetic regulation pathways of interest within each dataset and used 'pheatmap' [62] to evaluate if correlations were being driven by overlapping genes contained within the pathways.

\section{Horvath methylation age prediction}

The CpGs required for estimating the Horvath DNA methylation age [63] were derived from the published dataset and used in an $\mathrm{R}$ pipeline containing a modified version of Steve Horvath's code (https://dnamage.genetics.ucla.edu) to perform normalization and estimate the biological age in human TB published data [8]. The statistical tests were run using GraphPad Prism (version 9.1.0) for macOS. All-cause mortality was derived based on Horvath age estimates as described [27]; briefly they estimated that for every 5-year increase in Horvath age the Hazard increases by 1.14 . Increased risk was estimated by the formula: Increased Risk $=(($ Horvath age estimate $) / 5) \times 1.14$. The CI were multiplied by the same factor as the increased risk.

\section{RNA age prediction}

We sought publicly available RNA Sequencing data on the GEO. To be eligible for analysis, the data needed to contain whole blood samples from participants with active TB samples collected from time of diagnosis, prior to treatment, as well as patient level chronological age. We selected the largest such dataset for the RNA Age prediction, GSE107993 [26]. We removed outliners, patients missing chronological age, and only used baseline samples across all disease categories. RNA age was calculated using the 'RNAAgeCalc' package in R [64]. We calculated RNA age using the raw sequencing counts, specified that our tissue was 
blood, our signature type was 'all', and used the Peter's age signature. Considering the increase in biological age above chronological age as measured by DNA methylation, we calculated the difference between RNA and chronological age and used a one-way ANOVA with Tukey's multiple comparison to evaluate if the age difference was significantly increased with TB disease compared to household contacts, LTBI, and future LTBI progressors.

\section{Abbreviations}

DE: Differential expression; DHG: Differential hypermethylated genes; DMCs: Differentially methylated CpG sites; DMRs: Differentially methylated regions; DNA: Deoxyribonucleic acid; FC: Fold change; FDR: False discovery rate; GEO: Gene Expression Omnibus; GO: Gene Ontology; GSEA: Gene set enrichment analysis; HR: Hazard Ratio; HIV: Human Immuno-deficiency virus; LCMV: Lymphocytic choriomeningitis virus; LTBI: Latent Tuberculosis Infection; MSigDB: Molecular Signature Database; Mtb: Mycobacterium Tuberculosis; NCBI: National Center for Biotechnology Information; NES: Normalized enrichment scores; OS: Oxidative-stress; OSIS: Oxidative-stress induced senescence; PBMCs: Peripheral blood mononuclear cells; PCR: Polymerase chain reaction; RNA: Ribonucleic acid; RRBS: Representation bisulfite sequencing; SA-PE: Streptavidin-phycoerythrin; SASP: Senescence associated secretory pathway; TF: Transcription factors.

\section{AUTHOR CONTRIBUTIONS}

A.R.D and C.C. outlined the study design. K.K., T.N., and J.D.C. implemented the Cavia experiments. A., T.G., S.L.G., and C.C. implemented bioinformatic analysis of RRBS data. G.M., Q.D., J.K., S.C.L., T.N. and A. collected, implemented, and analyzed the multiplex ELISA experiments. A.R.D, T.G., S.L.G., T.N., and C.C. implemented the CD4, CD8, and CD14 human DNA methylation experiments. C.A.B. curated the gene expression array and RNA-seq datasets. C.A.B. and J.H. designed the gene expression analysis and C.A.B. conduced the gene expression analyses. C.A.B., H.N., J.H., and J.O. created and analyzed the enrichment map. C.A.B., A., S.L.G, C.C. and J.O. designed and conducted the statistical analyses. C.A.B., A., and S.L.G. prepared all manuscript figures. C.A.B., A., and A.R.D. completed the manuscript writing and all authors edited, revised, and approved the manuscript.

\section{CONFLICTS OF INTEREST}

The authors declare that they have no conflicts of interest.

\section{FUNDING}

ARD is supported by NIAID K23 AI141681-02. TG, SLG, and CC are partially supported by the Cancer Prevention Institute of Texas (CPRIT) RP170005, RP210227, and RP200504, NIH P30 shared resource grant CA125123, and NIEHS grants 1P30ES030285 and 1P42ES027725. JDC is funded in part from funds provided by the Texas A\&M University System and National Institutes of Health grants AI104960 and AI149383. JEH is supported by NHLBI R01 HL146228-01, the Cystic Fibrosis Foundation Hill18A0-CI, the National Science and Engineering Research Council, Canada, AWD-01777,3 and the National Health and Medical Research Council, Australia GNT1182929. CAB was supported by the Burroughs Wellcome Fund institutional program grant unifying population and laboratory-based sciences to Dartmouth College (Grant\#1014106).

\section{REFERENCES}

1. World Health Organization. Global Tuberculosis Report 2020. 2020; Geneva: World Health Organization.

2. Romanowski K, Baumann B, Basham CA, Ahmad Khan F, Fox GJ, Johnston JC. Long-term all-cause mortality in people treated for tuberculosis: a systematic review and meta-analysis. Lancet Infect Dis. 2019; 19:1129-37. https://doi.org/10.1016/S1473-3099(19)30309-3 PMID:31324519

3. Bello S, Afolabi RF, Ajayi DT, Sharma T, Owoeye DO, Oduyoye O, Jasanya J. Empirical evidence of delays in diagnosis and treatment of pulmonary tuberculosis: systematic review and meta-regression analysis. BMC Public Health. 2019; 19:820. https://doi.org/10.1186/s12889-019-7026-4 PMID:31238906

4. Lee-Rodriguez C, Wada PY, Hung YY, Skarbinski J. Association of Mortality and Years of Potential Life Lost With Active Tuberculosis in the United States. JAMA Netw Open. 2020; 3:e2014481. https://doi.org/10.1001/jamanetworkopen.2020.14481 PMID:32965497

5. Abhimanyu, Ontiveros CO, Guerra-Resendez RS, Nishiguchi T, Ladki M, Hilton IB, Schlesinger LS, DiNardo AR. Reversing Post-Infectious EpigeneticMediated Immune Suppression. Front Immunol. 2021; $12: 688132$. https://doi.org/10.3389/fimmu.2021.688132 PMID: $\underline{34163486}$

6. Vachharajani VT, Liu T, Brown CM, Wang X, Buechler NL, Wells JD, Yoza BK, McCall CE. SIRT1 inhibition during the hypoinflammatory phenotype of sepsis 
enhances immunity and improves outcome. J Leukoc Biol. 2014; 96:785-96. https://doi.org/10.1189/ilb.3MA0114-034RR PMID:25001863

7. DiNardo AR, Netea MG, Musher DM. Postinfectious Epigenetic Immune Modifications - A Double-Edged Sword. N Engl J Med. 2021; 384:261-70. https://doi.org/10.1056/NEJMra2028358 PMID:33471978

8. DiNardo AR, Rajapakshe K, Nishiguchi T, Grimm SL, Mtetwa G, Dlamini Q, Kahari J, Mahapatra S, Kay A, Maphalala G, Mace EM, Makedonas G, Cirillo JD, et al. DNA hypermethylation during tuberculosis dampens host immune responsiveness. J Clin Invest. 2020; 130:3113-23. https://doi.org/10.1172/JCl134622 PMID: 32125282

9. Roquilly $A$, Jacqueline $C$, Davieau $M$, Mollé $A$, Sadek $A$, Fourgeux $C$, Rooze $P$, Broquet $A$, Misme-Aucouturier B, Chaumette T, Vourc'h M, Cinotti R, Marec N, et al. Alveolar macrophages are epigenetically altered after inflammation, leading to long-term lung immunoparalysis. Nat Immunol. 2020; 21:636-48.

https://doi.org/10.1038/s41590-020-0673-x PMID:32424365

10. Ghoneim HE, Fan $Y$, Moustaki A, Abdelsamed HA, Dash P, Dogra P, Carter R, Awad W, Neale G, Thomas PG, Youngblood B. De Novo Epigenetic Programs Inhibit PD-1 Blockade-Mediated T Cell Rejuvenation. Cell. 2017; 170:142-57.e19. https://doi.org/10.1016/j.cell.2017.06.007 PMID:28648661

11. Sen DR, Kaminski J, Barnitz RA, Kurachi $M$, Gerdemann U, Yates KB, Tsao HW, Godec J, LaFleur MW, Brown FD, Tonnerre P, Chung RT, Tully DC, et al. The epigenetic landscape of $\mathrm{T}$ cell exhaustion. Science. 2016; 354:1165-9.

https://doi.org/10.1126/science.aae0491 PMID:27789799

12. Torres $K C L$, Rezende VB, Lima-Silva ML, Santos LJS, Costa CG, Mambrini JVM, Peixoto SV, TarazonaSantos E, Martins Filho OA, Lima-Costa MF, TeixeiraCarvalho A. Immune senescence and biomarkers profile of Bambuí aged population-based cohort. Exp Gerontol. 2018; 103:47-56.

https://doi.org/10.1016/i.exger.2017.12.006 PMID:29247791

13. McMurray DN. Disease model: pulmonary tuberculosis. Trends Mol Med. 2001; 7:135-7. https://doi.org/10.1016/s1471-4914(00)01901-8 PMID: $\underline{11286786}$

14. Pereira RM, Hogan PG, Rao A, Martinez GJ. Transcriptional and epigenetic regulation of $T$ cell hyporesponsiveness. J Leukoc Biol. 2017; 102:601-15.
https://doi.org/10.1189/jlb.2RI0317-097R PMID:28606939

15. Zhou Y, Zhou B, Pache L, Chang M, Khodabakhshi AH, Tanaseichuk O, Benner C, Chanda SK. Metascape provides a biologist-oriented resource for the analysis of systems-level datasets. Nat Commun. 2019; 10:1523.

https://doi.org/10.1038/s41467-019-09234-6 PMID:30944313

16. Bloom Cl, Graham CM, Berry MP, Rozakeas F, Redford PS, Wang $Y, X u Z$, Wilkinson KA, Wilkinson RJ, Kendrick $Y$, Devouassoux $G$, Ferry $T$, Miyara $M$, et al. Transcriptional blood signatures distinguish pulmonary tuberculosis, pulmonary sarcoidosis, pneumonias and lung cancers. PLoS One. 2013; 8:e70630.

https://doi.org/10.1371/journal.pone.0070630 PMID:23940611

17. Kaforou M, Wright VJ, Oni T, French N, Anderson ST, Bangani N, Banwell CM, Brent AJ, Crampin AC, Dockrell HM, Eley B, Heyderman RS, Hibberd ML, et al. Detection of tuberculosis in HIV-infected and uninfected African adults using whole blood RNA expression signatures: a case-control study. PLOS Med. 2013; 10:e1001538.

https://doi.org/10.1371/journal.pmed.1001538 PMID:24167453

18. Anderson ST, Kaforou M, Brent AJ, Wright VJ, Banwell CM, Chagaluka G, Crampin AC, Dockrell HM, French $\mathrm{N}$, Hamilton MS, Hibberd ML, Kern F, Langford PR, et al. Diagnosis of childhood tuberculosis and host RNA expression in Africa. N Engl J Med. 2014; 370:171223.

https://doi.org/10.1056/NEJMoa1303657 PMID:24785206

19. Bobak CA, Abbolish TB. Analysis of Blood and Breath' Omics to Lend Insights and Strategies for Hindering Tuberculosis. 2021.

20. Lu AT, Quach A, Wilson JG, Reiner AP, Aviv A, Raj K, Hou L, Baccarelli AA, Li Y, Stewart JD, Whitsel EA, Assimes TL, Ferrucci L, Horvath S. DNA methylation GrimAge strongly predicts lifespan and healthspan. Aging (Albany NY). 2019; 11:303-27.

https://doi.org/10.18632/aging.101684 PMID:30669119

21. Ucar D, Márquez EJ, Chung CH, Marches R, Rossi RJ, Uyar A, Wu TC, George J, Stitzel ML, Palucka AK, Kuchel GA, Banchereau J. The chromatin accessibility signature of human immune aging stems from $\mathrm{CD}^{+} \mathrm{T}$ cells. J Exp Med. 2017; 214:3123-44.

https://doi.org/10.1084/jem.20170416 PMID:28904110 
22. Mahalingaiah PK, Ponnusamy L, Singh KP. Oxidative stress-induced epigenetic changes associated with malignant transformation of human kidney epithelial cells. Oncotarget. 2017; 8:11127-43.

https://doi.org/10.18632/oncotarget.12091 PMID:27655674

23. Vrtačnik P, Zupan J, Mlakar V, Kranjc T, Marc J, Kern B, Ostanek B. Epigenetic enzymes influenced by oxidative stress and hypoxia mimetic in osteoblasts are differentially expressed in patients with osteoporosis and osteoarthritis. Sci Rep. 2018; 8:16215.

https://doi.org/10.1038/s41598-018-34255-4 PMID: 30385847

24. Horvath S, Levine AJ. HIV-1 Infection Accelerates Age According to the Epigenetic Clock. J Infect Dis. 2015; 212:1563-73.

https://doi.org/10.1093/infdis/jiv277 PMID:25969563

25. Gross AM, Jaeger PA, Kreisberg JF, Licon $K$, Jepsen $K L$, Khosroheidari M, Morsey BM, Swindells S, Shen H, Ng CT, Flagg K, Chen D, Zhang K, et al. Methylome-wide Analysis of Chronic HIV Infection Reveals Five-Year Increase in Biological Age and Epigenetic Targeting of HLA. Mol Cell. 2016; 62:157-68.

https://doi.org/10.1016/i.molcel.2016.03.019 PMID:27105112

26. Singhania A, Verma R, Graham CM, Lee J, Tran T, Richardson $M$, Lecine $P$, Leissner $P$, Berry MPR, Wilkinson RJ, Kaiser K, Rodrigue M, Woltmann G, et al. A modular transcriptional signature identifies phenotypic heterogeneity of human tuberculosis infection. Nat Commun. 2018; 9:2308.

https://doi.org/10.1038/s41467-018-04579-w PMID:29921861

27. Zhang $Y$, Wilson $R$, Heiss J, Breitling LP, Saum KU, Schöttker B, Holleczek B, Waldenberger M, Peters A, Brenner $\mathrm{H}$. DNA methylation signatures in peripheral blood strongly predict all-cause mortality. Nat Commun. 2017; 8:14617.

https://doi.org/10.1038/ncomms14617 PMID:28303888

28. Wen H, Dou Y, Hogaboam CM, Kunkel SL. Epigenetic regulation of dendritic cell-derived interleukin-12 facilitates immunosuppression after a severe innate immune response. Blood. 2008; 111:1797-804. https://doi.org/10.1182/blood-2007-08-106443 PMID: 18055863

29. Kathirvel M, Mahadevan S. The role of epigenetics in tuberculosis infection. Epigenomics. 2016; 8:537-49. https://doi.org/10.2217/epi.16.1 PMID:27035266

30. Pennini ME, Pai RK, Schultz DC, Boom WH, Harding CV. Mycobacterium tuberculosis 19-kDa lipoprotein inhibits IFN-gamma-induced chromatin remodeling of MHC2TA by TLR2 and MAPK signaling. J Immunol. 2006; 176:4323-30.

https://doi.org/10.4049/jimmunol.176.7.4323 PMID:16547269

31. Yaseen I, Kaur P, Nandicoori VK, Khosla S. Mycobacteria modulate host epigenetic machinery by Rv1988 methylation of a non-tail arginine of histone H3. Nat Commun. 2015; 6:8922.

https://doi.org/10.1038/ncomms9922

PMID:26568365

32. Mikovits JA, Young HA, Vertino $P$, Issa JP, Pitha PM, Turcoski-Corrales S, Taub DD, Petrow CL, Baylin SB, Ruscetti FW. Infection with human immunodeficiency virus type 1 upregulates DNA methyltransferase, resulting in de novo methylation of the gamma interferon (IFN-gamma) promoter and subsequent downregulation of IFN-gamma production. Mol Cell Biol. 1998; 18:5166-77.

https://doi.org/10.1128/MCB.18.9.5166 PMID:9710601

33. Youngblood B, Noto A, Porichis F, Akondy RS, Ndhlovu ZM, Austin JW, Bordi R, Procopio FA, Miura T, Allen TM, Sidney J, Sette A, Walker BD, et al. Cutting edge: Prolonged exposure to HIV reinforces a poised epigenetic program for PD-1 expression in virusspecific CD8 T cells. J Immunol. 2013; 191:540-4. https://doi.org/10.4049/jimmunol.1203161 PMID:23772031

34. DiNardo AR, Nishiguchi T, Mace EM, Rajapakshe $K$, Mtetwa G, Kay A, Maphalala G, Secor WE, Mejia R, Orange JS, Coarfa C, Bhalla KN, Graviss EA, et al. Schistosomiasis Induces Persistent DNA Methylation and Tuberculosis-Specific Immune Changes. J Immunol. 2018; 201:124-33.

https://doi.org/10.4049/jimmunol.1800101 PMID:29752313

35. Prescott HC, Osterholzer JJ, Langa KM, Angus DC, Iwashyna TJ. Late mortality after sepsis: propensity matched cohort study. BMJ. 2016; 353:i2375.

https://doi.org/doi:10.1136/bmj.i2375

PMID:27189000

36. Sandvall B, Rueda AM, Musher DM. Long-term survival following pneumococcal pneumonia. Clin Infect Dis. 2013; 56:1145-6.

https://doi.org/10.1093/cid/cis1207

PMID:23300240

37. de Perio MA, Tsevat J, Roselle GA, Kralovic SM, Eckman $\mathrm{MH}$. Cost-effectiveness of interferon gamma release assays vs tuberculin skin tests in health care workers. Arch Intern Med. 2009; 169:179-87.

https://doi.org/10.1001/archinternmed.2008.524 PMID:19171815 
38. Freimane L, Barkane L, Igumnova V, Kivrane A, Zole E, Ranka R. Telomere length and mitochondrial DNA copy number in multidrug-resistant tuberculosis. Tuberculosis (Edinb). 2021; 131:102144. https://doi.org/10.1016/j.tube.2021.102144 PMID: $\underline{34781086}$

39. Martinez GJ, Pereira RM, Äijö T, Kim EY, Marangoni F, Pipkin ME, Togher S, Heissmeyer V, Zhang YC, Crotty S, Lamperti ED, Ansel KM, Mempel TR, et al. The transcription factor NFAT promotes exhaustion of activated $\mathrm{CD}^{+} \mathrm{T}$ cells. Immunity. 2015; 42:265-78. https://doi.org/10.1016/i.immuni.2015.01.006 PMID:25680272

40. Scott-Browne JP, López-Moyado IF, Trifari S, Wong V, Chavez L, Rao A, Pereira RM. Dynamic Changes in Chromatin Accessibility Occur in CD8 ${ }^{+} \mathrm{T}$ Cells Responding to Viral Infection. Immunity. 2016; 45:1327-40.

https://doi.org/10.1016/i.immuni.2016.10.028 PMID:27939672

41. Yang $\mathrm{HJ}$, Kong $\mathrm{Y}$, Cheng $\mathrm{Y}$, Janagama $\mathrm{H}$, Hassounah $\mathrm{H}$, Xie H, Rao J, Cirillo JD. Real-time Imaging of Mycobacterium tuberculosis, Using a Novel NearInfrared Fluorescent Substrate. J Infect Dis. 2017; 215:405-14.

https://doi.org/10.1093/infdis/iiw298

PMID:27421748

42. Sule $P$, Tilvawala $R$, Mustapha $T$, Hassounah $H$, Noormohamed A, Kundu S, Graviss EA, Walkup GK, Kong Y, Cirillo JD. Rapid Tuberculosis Diagnosis Using Reporter Enzyme Fluorescence. J Clin Microbiol. 2019; 57:e01462-19.

https://doi.org/10.1128/JCM.01462-19

PMID:31511338

43. Christensen LM, Sule P, Cirillo SLG, Strain M, Plumlee Q, Adams LG, Cirillo JD. Legionnaires' Disease Mortality in Guinea Pigs Involves the p45 Mobile Genomic Element. J Infect Dis. 2019; 220:1700-10.

https://doi.org/10.1093/infdis/jiz340

PMID:31268152

44. Gu H, Smith ZD, Bock C, Boyle P, Gnirke A, Meissner A. Preparation of reduced representation bisulfite sequencing libraries for genome-scale DNA methylation profiling. Nat Protoc. 2011; 6:468-81. https://doi.org/10.1038/nprot.2010.190 PMID:21412275

45. Meissner A, Mikkelsen TS, Gu H, Wernig M, Hanna J, Sivachenko A, Zhang $X$, Bernstein BE, Nusbaum C, Jaffe DB, Gnirke A, Jaenisch R, Lander ES. Genomescale DNA methylation maps of pluripotent and differentiated cells. Nature. 2008; 454:766-70.

https://doi.org/10.1038/nature07107

PMID: $\underline{18600261}$
46. Krueger F, Andrews SR. Bismark: a flexible aligner and methylation caller for Bisulfite-Seq applications. Bioinformatics. 2011; 27:1571-2.

https://doi.org/10.1093/bioinformatics/btr167 PMID:21493656

47. Park Y, Wu H. Differential methylation analysis for BSseq data under general experimental design. Bioinformatics. 2016; 32:1446-53.

https://doi.org/10.1093/bioinformatics/btw026 PMID:26819470

48. Quinlan AR, Hall IM. BEDTools: a flexible suite of utilities for comparing genomic features. Bioinformatics. 2010; 26:841-2.

https://doi.org/10.1093/bioinformatics/btq033 PMID:20110278

49. Liberzon A, Birger C, Thorvaldsdóttir $H$, Ghandi $M$, Mesirov JP, Tamayo P. The Molecular Signatures Database (MSigDB) hallmark gene set collection. Cell Syst. 2015; 1:417-25.

https://doi.org/10.1016/i.cels.2015.12.004 PMID:26771021

50. Shannon P, Markiel A, Ozier O, Baliga NS, Wang JT, Ramage D, Amin N, Schwikowski B, Ideker T. Cytoscape: a software environment for integrated models of biomolecular interaction networks. Genome Res. 2003; 13:2498-504.

https://doi.org/10.1101/gr.1239303

PMID:14597658

51. Bader GD, Hogue CW. An automated method for finding molecular complexes in large protein interaction networks. BMC Bioinformatics. 2003; 4:2. https://doi.org/10.1186/1471-2105-4-2 PMID: $\underline{12525261}$

52. Swift ML. GraphPad Prism, Data Analysis, and Scientific Graphing. J Chem Inf Comput Sci. 1997; 37:411-2.

53. Coppé JP, Patil CK, Rodier F, Sun Y, Muñoz DP, Goldstein J, Nelson PS, Desprez PY, Campisi J. Senescence-associated secretory phenotypes reveal cell-nonautonomous functions of oncogenic RAS and the p53 tumor suppressor. PLoS Biol. 2008; 6:2853-68. https://doi.org/10.1371/journal.pbio.0060301 PMID:19053174

54. Lasry A, Ben-Neriah Y. Senescence-associated inflammatory responses: aging and cancer perspectives. Trends Immunol. 2015; 36:217-28. https://doi.org/10.1016/i.it.2015.02.009 PMID:25801910

55. Davis S, Meltzer PS. GEOquery: a bridge between the Gene Expression Omnibus (GEO) and BioConductor. Bioinformatics. 2007; 23:1846-7. https://doi.org/10.1093/bioinformatics/btm254 PMID: $\underline{17496320}$ 
56. Plaisier SB, Taschereau R, Wong JA, Graeber TG. Rank-rank hypergeometric overlap: identification of statistically significant overlap between geneexpression signatures. Nucleic Acids Res. 2010; 38:e169.

https://doi.org/10.1093/nar/gkq636

PMID:20660011

57. Subramanian A, Tamayo P, Mootha VK, Mukherjee S, Ebert BL, Gillette MA, Paulovich A, Pomeroy SL, Golub TR, Lander ES, Mesirov JP. Gene set enrichment analysis: a knowledge-based approach for interpreting genome-wide expression profiles. Proc Natl Acad Sci U S A. 2005; 102:15545-50. https://doi.org/10.1073/pnas.0506580102 PMID:16199517

58. Merico D, Isserlin R, Stueker O, Emili A, Bader GD. Enrichment map: a network-based method for geneset enrichment visualization and interpretation. PLoS One. 2010; 5:e13984. https://doi.org/10.1371/journal.pone.0013984 PMID:21085593

59. Kucera $M$, Isserlin $R$, Arkhangorodsky A, Bader GD. AutoAnnotate: A Cytoscape app for summarizing networks with semantic annotations. F1000Res. 2016; 5:1717.

https://doi.org/10.12688/f1000research.9090.1

PMID:27830058
60. Sweeney TE, Braviak L, Tato CM, Khatri P. Genomewide expression for diagnosis of pulmonary tuberculosis: a multicohort analysis. Lancet Respir Med. 2016; 4:213-24.

https://doi.org/10.1016/S2213-2600(16)00048-5 PMID:26907218

61. Wilcoxon F. Individual Comparisons by Ranking Methods. Biometrics Bulletin. 1945; 1:80-3. https://doi.org/10.2307/3001968"

62. Kolde R. Pretty Heatmaps. 2018.

63. Horvath S. DNA methylation age of human tissues and cell types. Genome Biol. 2013; 14:R115. https://doi.org/10.1186/gb-2013-14-10-r115 PMID:24138928

64. Ren X, Kuan PF. RNAAgeCalc: A multi-tissue transcriptional age calculator. PLoS One. 2020; 15:e0237006.

https://doi.org/10.1371/journal.pone.0237006 PMID:32750074 


\section{SUPPLEMENTARY MATERIALS}

\section{Supplementary Figures}

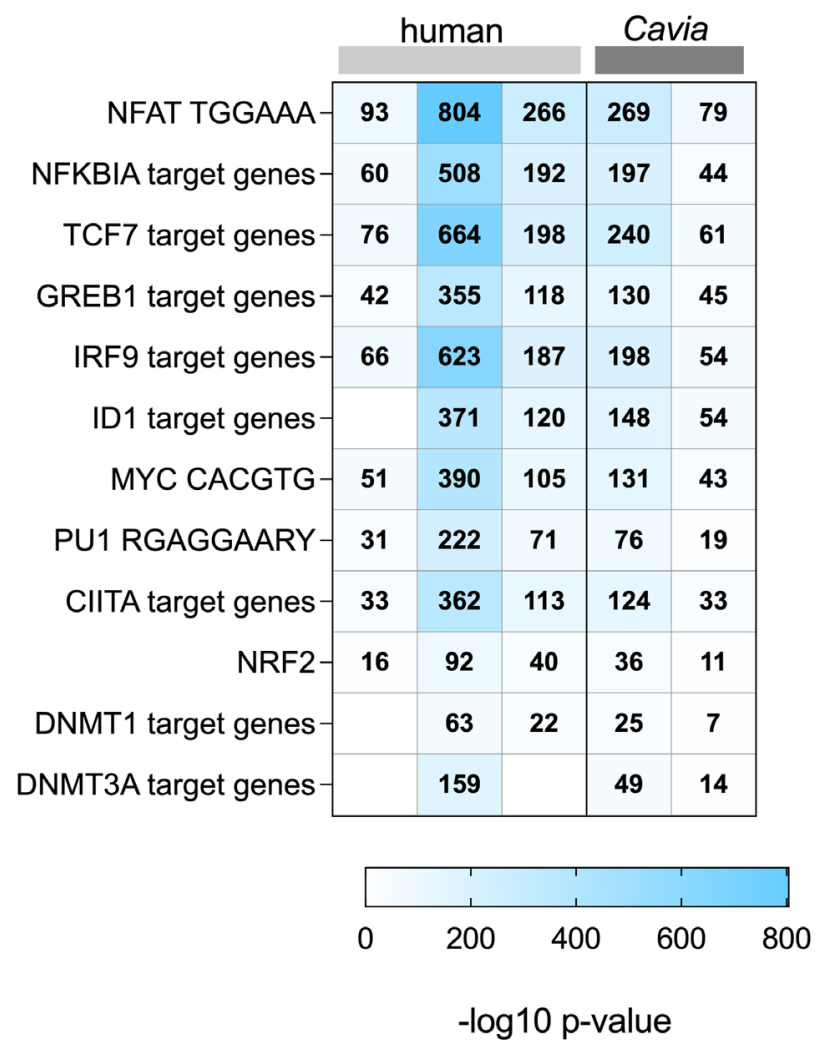

Supplementary Figure 1. TF overlap between guinea pigs and humans. MsigDB Gene set enrichment analysis shows considerable overlap in TF targets between humans (light grey bars) and guinea pigs (dark grey bars) with TB. The box colors show -log $10 p$-value of enrichment, with darker shades of blue indicating significance. The numbers in the squares indicate the predicted gene count. Only a subset of TB-relevant TF targets are shown, for the full list refer to Supplementary Table 3. 

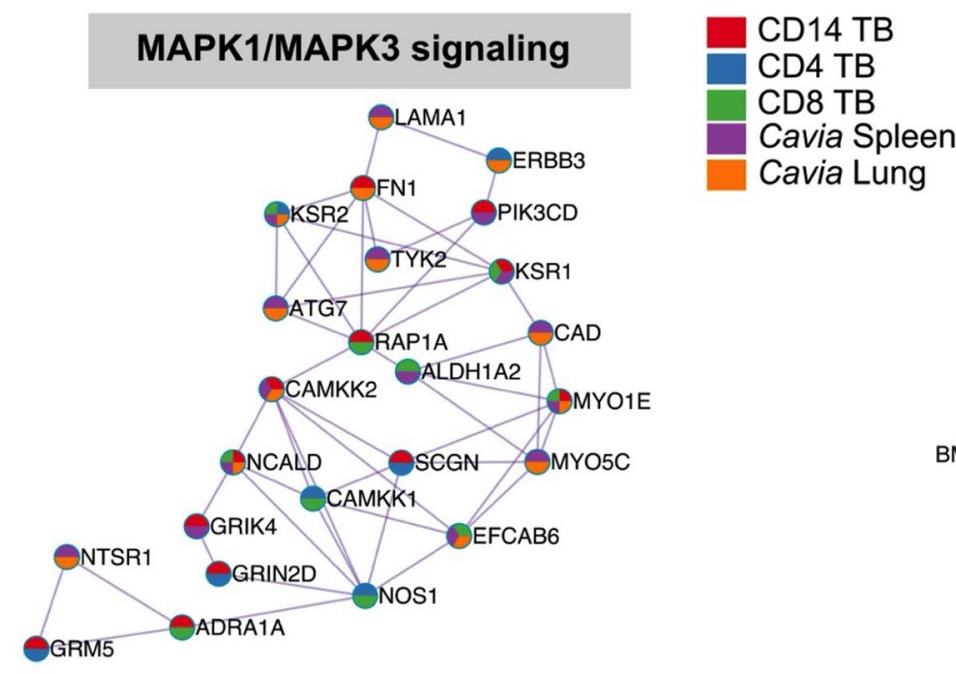

\section{Endocytosis}

Cavia Lung

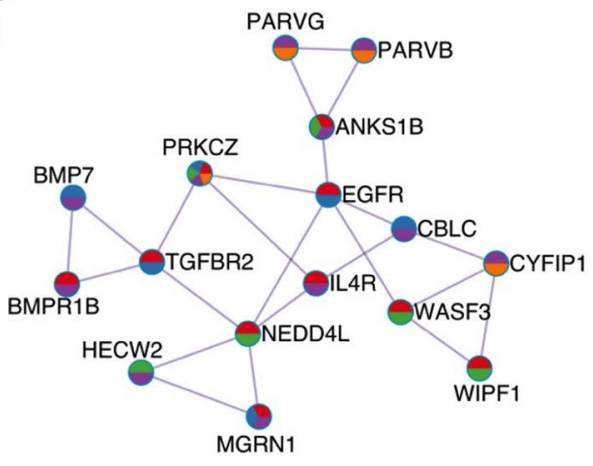

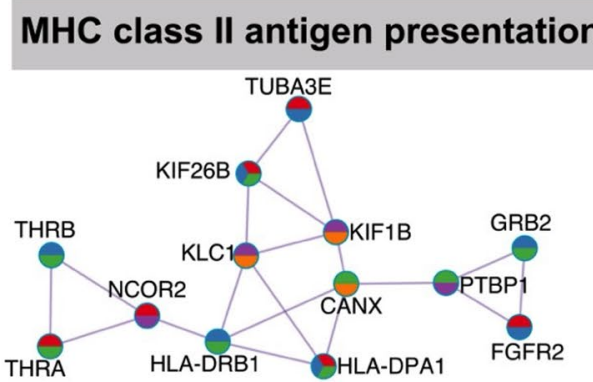

Calcium ion transport

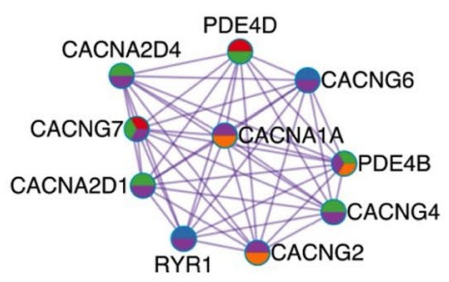

\section{Cell adhesion molecules}

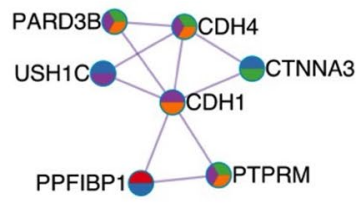

\section{O-glycosylation of proteins}

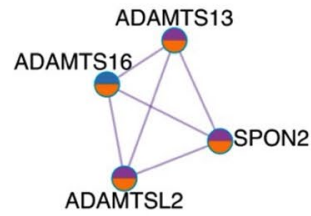

\section{Anti-inflammatory response}

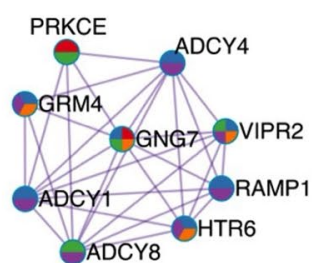

\section{Chromatin modifying enzymes}

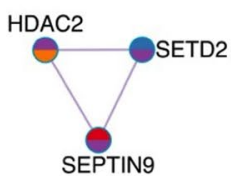

Supplementary Figure 2. Shared genes and pathway between guinea pigs and humans. Enrichment network generated in Metascape [15] from five gene lists, three from human data [8] and two from Cavia generated in this study. Top 1000 differentially hypermethylated genes from all merged gene list was used for analysis. The nodes represent pie charts, where the color represents the identity in the input gene list. Cluster labels are derived from functional enriched gene term for that cluster. If the same gene is shared between multiple lists, the color appears in the pie. 

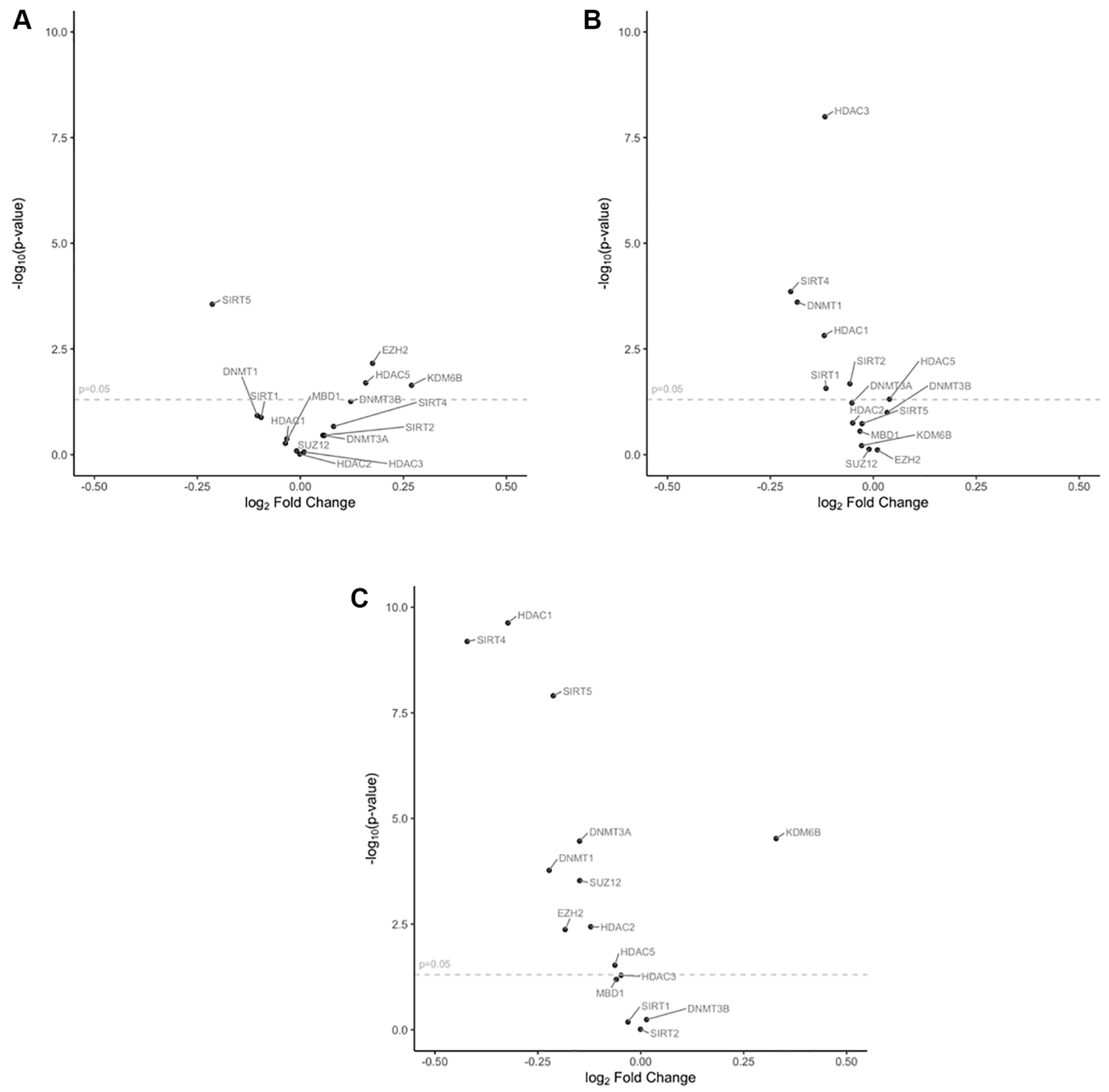

Supplementary Figure 3. Volcano plots showing the differential gene expression of canonical genes associated with epigenetic regulation in (A) GSE42834, (B) GSE37250, and (C) GSE39940. Differential expression (DE) analysis of genes was conducted using the 'limma' package in R to calculate the $\log _{2} \mathrm{FC}$ and $p$-value of each gene where active TB was contrasted to all the other disease classes as a one-vs.-rest problem. 
A
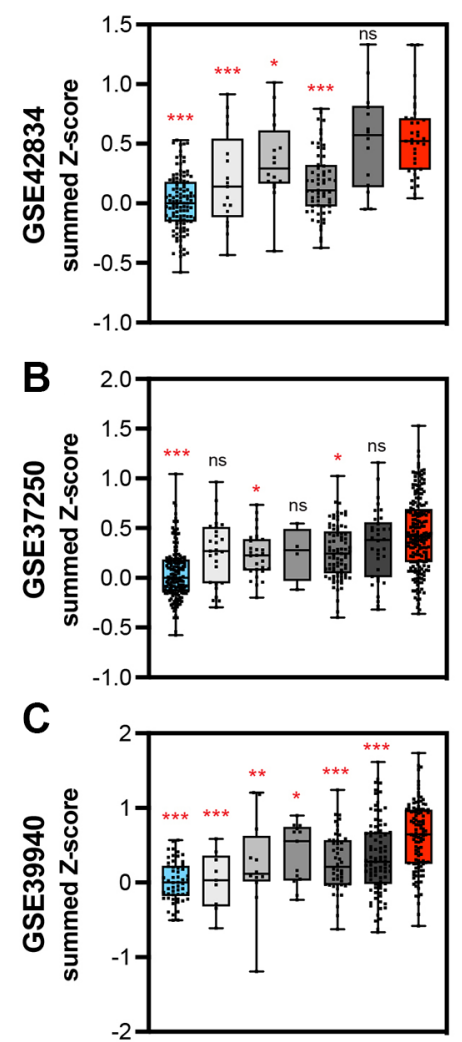

Senescence-Associated Secretory Phenotype (SASP
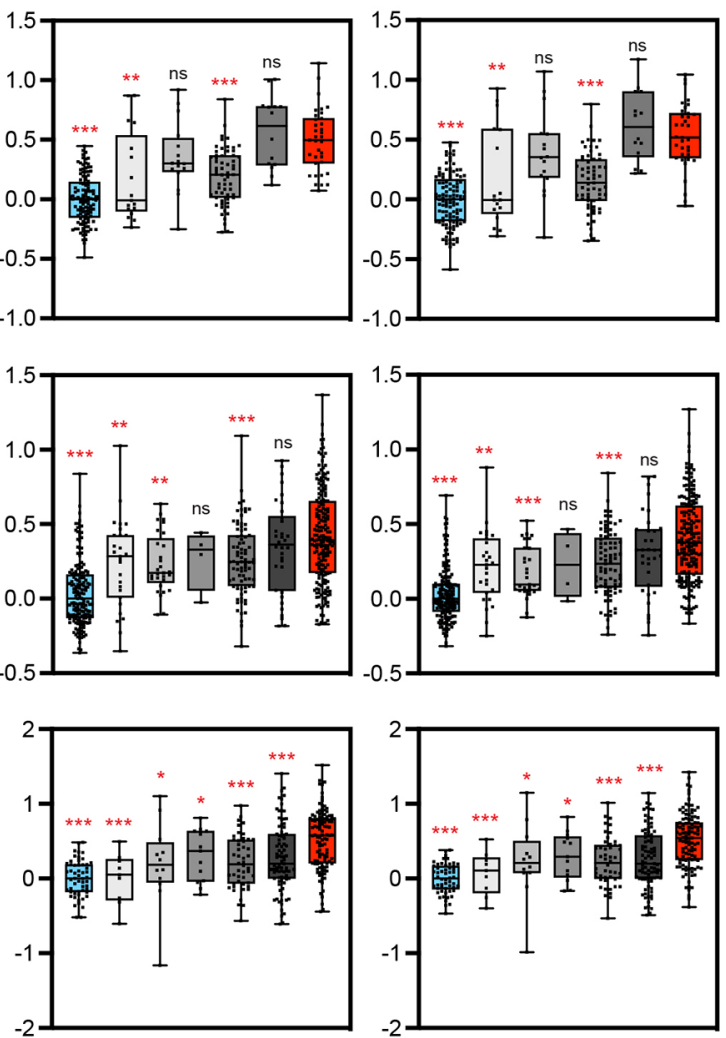

Oxidative Stress-Induced Senescence (OSIS)
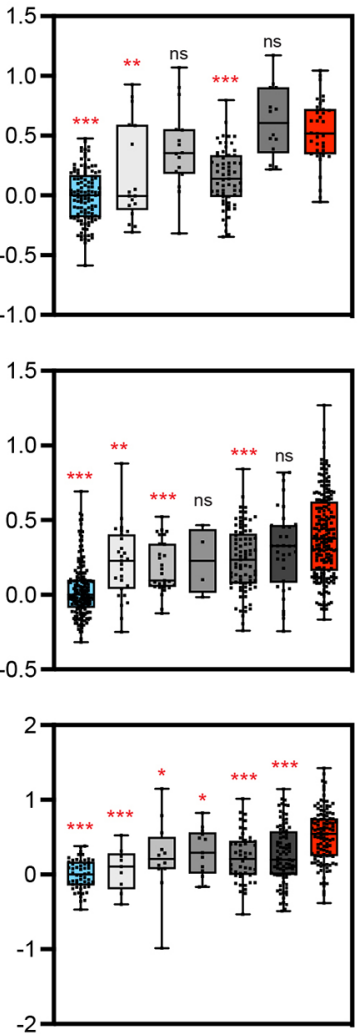

Healthy control $(n=113)$

Treated other disease $(n=17)$

$\square$ Cancer $(n=16)$

$\square$ Sarcoidosis $(n=61)$

$\square$ Pneumonia $(n=14)$

${ }^{*} p<0.05$

** $p<0.01$

*** $p<0.001$

TB $(n=35)$

Asymptomatic TB Infection (LTBI) $(n=54)$

Other disease $(n=9)$

Cancer $(n=12)$

Chronic infection $(n=13)$

Acute infection $(n=48)$

Pneumonia $(n=87)$

TB $(n=111)$

Supplementary Figure 4. DNA methylation and cellular senescence genes are increased TB and other chronic infections. (A-C) A summed z-score for gene expression from each patient was assessed for pathways including DNA methylation, SASP, and OSIS pathways, with all three studies demonstrating increased summed z-scores in TB patients (red box plot) as compared to other groups. $P$-values from a Wilcoxon rank sum test are indicated by asterisks. Abbreviations: SASP: Senescence-associated secretory phenotype; OSIS: Oxidative stress-induced senescence. 


\section{Supplementary Tables}

Please browse Full Text version to see the data of Supplementary Tables 1-3.

Supplementary Table 1. DNA methylation results in the guinea pig (Cavia Porcellus) model of TB infection.

Supplementary Table 2. Pathway analysis of hypermethylated genes in humans and guinea pig (Cavia Porcellus) with TB.

Supplementary Table 3. Epigenetic changes in genes targeted by immune-related transcription factors (TF) in humans and guinea pig (Cavia Porcellus) with TB. 